\title{
Infective Endocarditis-Associated Glomerulonephritis: A Comprehensive Review of the Clinical Presentation, Histopathology, and Management
}

\author{
Tomoaki Takata,* Yukari Mae,* Takaaki Sugihara* and Hajime Isomoto* \\ *Division of Gastroenterology and Nephrology, Department of Multidisciplinary Internal Medicine, School of Medicine, Faculty of \\ Medicine, Tottori University, Yonago 683-8504, Japan
}

\begin{abstract}
A significant proportion of patients with infective endocarditis presents with acute renal failure related to infective endocarditis-associated glomerulonephritis (IEAGN). However, the clinical presentation of IEAGN differs from that of other infection-related glomerulonephritis (IRGN) with anti-neutrophil cytoplasmic antibody (ANCA) positivity occurring in almost onethird of cases; therefore, it may be difficult to establish a definitive diagnosis and provide appropriate treatment. This review article provides a comprehensive understanding of the clinical presentation, investigations, histopathology, and treatment/management of IEAGN so that clinicians can keep this differential in mind for patients with fever of unknown origin accompanied by signs and symptoms of acute renal failure.
\end{abstract}

Key words acute kidney injury; anti-neutrophil cytoplasmic antibody; infection-related glomerulonephritis; infective endocarditis-associated glomerulonephritis; rapidly progressive glomerulonephritis

It is a well-established fact that glomerulonephritis can accompany bacterial infections. In the past, acute glomerulonephritis typically developed after group A beta-hemolytic streptococcal infection; however, the incidence of glomerulonephritis due to Staphylococcus aureus infection is increasing. ${ }^{1}$ The number of cases of glomerulonephritis associated with infective endocarditis reported in the literature is also increasing. The clinical presentation of glomerulonephritis associated with infective endocarditis is different from that of conventional acute poststreptococcal glomerulonephritis (APSGN). In contrast with the typical presentation of ASPGN, bacterial infectionassociated glomerulonephritis may develop even before

Corresponding author: Tomoaki Takata, MD, PhD

t-takata@tottori-u.ac.jp

Received 2021 October 19

Accepted 2021 December 28

Online published 2022 January 28

Abbreviations: ANCA, anti-neutrophil cytoplasmic antibodies; APSGN, acute poststreptococcal glomerulonephritis; IEAGN, infective endocarditis-associated glomerulonephritis; IRGN, infection-related glomerulonephritis the resolution of infection. Therefore, "infection-related glomerulonephritis" (IRGN) has been proposed as a collective term for glomerulonephritis that occurs due to an infection, as this term describes the pathogenesis of glomerulonephritis more accurately. ${ }^{2}$ Infection related glomerulonephritis includes glomerulonephritis caused by bacteria such as Streptococcus and Staphylococcus, viruses and other causative pathogens (Fig. 1). Although infective endocarditis-associated glomerulonephritis (IEAGN) is a disease group that is included in IRGN, it is not as easily diagnosed as APSGN, because the presence of infection needs to be confirmed by culture; and the focus of infection is identified using imaging, such as an echocardiography. In addition, there are cases of IEAGN that are positive for anti-neutrophil cytoplasmic antibodies (ANCA), which need to be taken into account before establishing a diagnosis. Further, although IEAGN may be similar to glomerulonephritis associated with Staphylococcus in terms of pathogenic bacteria, differences in their clinical features have also been reported. In this review, we summarize the clinical features, points of differentiation, and other aspects of IEAGN, the number of case reports for which have increased in recent years.

\section{EPIDEMIOLOGY}

Renal lesions complicating infective endocarditis have been well-known for a long time, starting with a series of seven cases reported by Löhlein et al. in $1910 .^{3-5}$ Infective endocarditis is usually diagnosed based on the Duke's criteria, ${ }^{6}$ which include pathological criteria for detecting the pathogenic microorganism and confirming the presence of endocarditis, and clinical criteria which require blood cultures, echocardiogram findings, and clinical symptoms. The diagnosis is made if the patient has the required number of positive findings, including pathological evidence of pathogenic microorganism or infective endocarditis, repeat positive blood culture, ultrasound findings indicative of infective endocarditis, and newly occurring valve regurgitation. ${ }^{7,8}$

Infective endocarditis is classically divided into two categories: subacute and acute, which differ on the basis of causative microorganisms, clinical presentation, and patient characteristics. Subacute endocarditis 


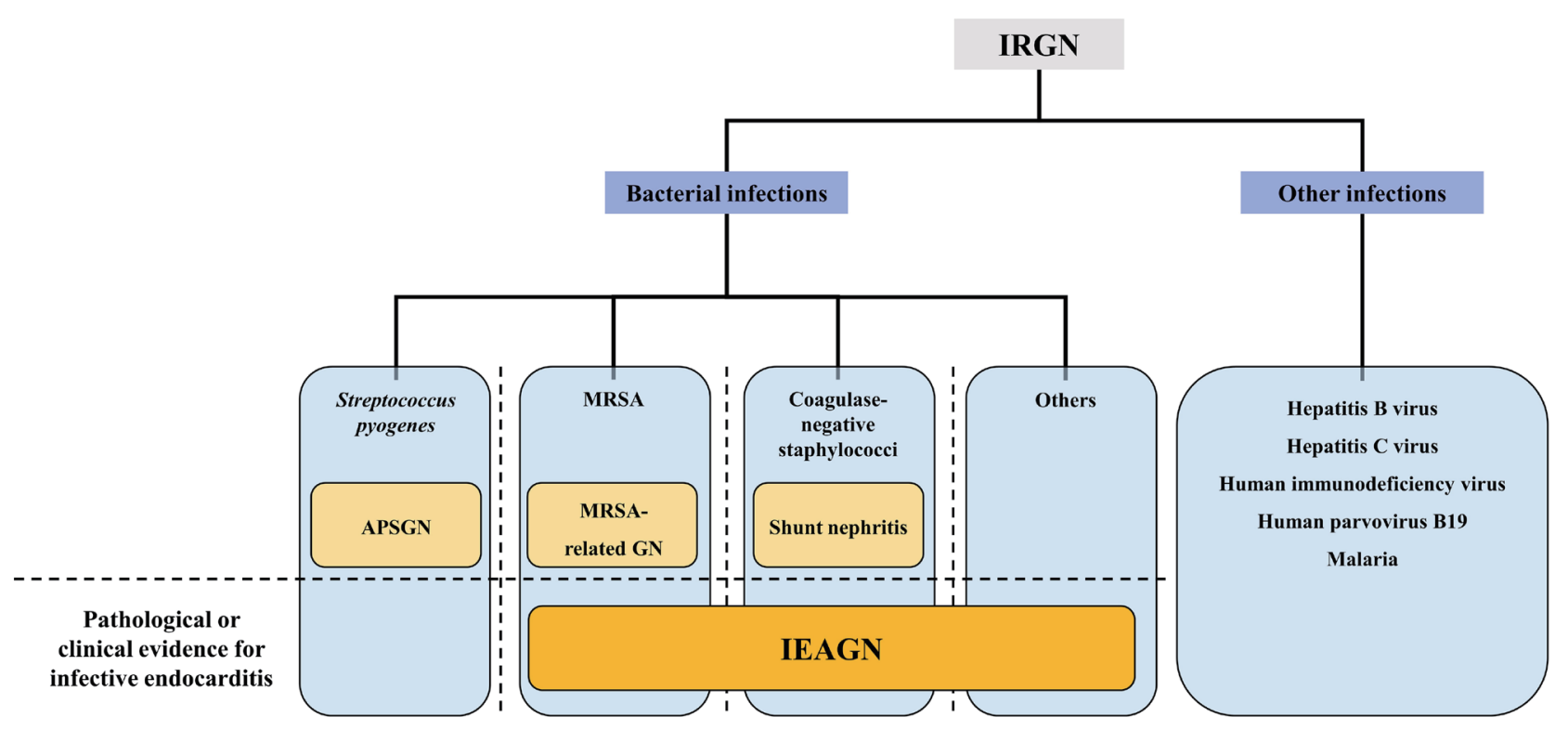

Fig. 1. Schematic for the classification of infection-related glomerulonephritis. Bacterial infection-related glomerulonephritis mainly refers to APSGN, MRSA-related GN, shunt nephritis, and IEAGN. APSGN, acute post-streptococcal glomerulonephritis; IEAGN, infective endocarditis-related glomerulonephritis; IRGN, infection-related glomerulonephritis; MRSA, methicilin resistant Staphylococcus aureus.

usually presents with low-grade fever and mild heart failure symptoms, and often has a chronic course. It is usually caused by less virulent bacteria such as Streptococcus mutans, Streptococcus viridans, and the HACEK group (Haemophilus spp., Aggregatibacter spp., Cardiobacterium hominis, Eikenella corrodens, Kingella spp.). In contrast to subacute infective endocarditis, cases caused by strongly histoclastic bacteria such as Staphylococcus aureus often follow an acute course. ${ }^{8}$

Patient risk factors for in subacute infective endocarditis include underlying congenital heart disease or valvular disease accompanying rheumatic fever. ${ }^{9}$ Risk factors for acute infective endocarditis include intravenous drug use, diabetes, malignancies, and other pathologies that can cause immunosuppression. ${ }^{8}$ There has also been an increase in healthcare-related cases, such as those associated with valve replacement, pacemakers, implantable defibrillators, and long-term intravascular catheters. A study by Benito et al, found that $19 \%$ of infective endocarditis cases (303 out of 1622 cases) were caused by nosocomial infections. ${ }^{10}$ The patient cohort in that report consisted of patients with definite native valve endocarditis and no history of injection drug use recruited from 61 facilities in 28 countries from 2000 to 2005. Moreover, in about half of the healthcare-associated infective endocarditis cases, Staphylococcus aureus was the causative organism.

One study found that one-third of the cases were complicated by acute renal failure. ${ }^{11}$ Since glomerulonephritis is an important cause of acute renal failure, it is assumed that a significant proportion of patients with infective endocarditis develop acute renal failure due to glomerulonephritis. Meanwhile, IEAGN is reported to account for $6-20 \%$ cases of IRGN. ${ }^{12-15}$

\section{CLINICAL PRESENTATION}

According to Nasr et al., the diagnostic criteria for IRGN are met when at least three of the following are present: (1) presence of infectious disease before or at the onset of glomerulonephritis, (2) hypocomplementemia, (3) endocapillary proliferative and exudative glomerulonephritis on optical microscopy of renal biopsy tissue, (4) C3-dominant deposits on immunofluorescence, and (5) presence of hump-like subepithelial deposits on electron microscopy. ${ }^{1}$ Whether the same diagnostic criteria can be applied to IEAGN is still a topic of debate. However, because it is considered a type of IRGN, verifying the presence of infective endocarditis needs to be included in these criteria if they are being used to diagnose IEAGN.

As discussed above, infective endocarditis is a heterogeneous disease, with differing onset patterns depending on the causative organism and patient characteristics. However, incidence of subacute infective endocarditis has been declining thanks to recent developments in antibiotic therapies. In some cases, renal 
Table 1. Comparison of clinical characteristics of ASPGN and IEAGN $8,9,16,23,28$

\begin{tabular}{|c|c|c|}
\hline & APSGN & IEAGN \\
\hline Age & Mainly pediatric age & Mainly adults, particularly the elderly \\
\hline Host background & & $\begin{array}{l}\text { diabetes mellitus }^{9} \\
\text { Immunocompromised } \\
\text { Indwelling devices } \\
\text { Valvular disease }^{8}\end{array}$ \\
\hline Causative bacterium & Group A Streptococcus & $\begin{array}{l}\text { MRSA } \\
\text { viridans Streptococcus } \\
\text { HACEK group }\end{array}$ \\
\hline Latent period & $\begin{array}{l}1 \text { to } 2 \text { weeks after adenoiditis } \\
3 \text { to } 6 \text { weeks after skin infection }\end{array}$ & No infection-free latent period \\
\hline Clinical presentation & $\begin{array}{l}\text { Edema and hypertension } \\
\text { Proteinuria and hematuria } \\
\text { Normal to mild renal dysfunction }\end{array}$ & $\begin{array}{l}\text { Acute renal failure } \\
\text { Anemia and thrombopenia } \\
\text { Purpura }\end{array}$ \\
\hline Laboratory findings & $\begin{array}{l}\text { Low C3 level } \\
\text { Elevated ASO titer }\end{array}$ & $\begin{array}{l}\text { Low C3 in } 50 \% \text { of patients }{ }^{16} \\
\text { Positive ANCA in some patients }\end{array}$ \\
\hline Treatment and outcome & $\begin{array}{l}\text { Usually complete recovery without } \\
\text { immunosuppressive therapy or antibiotics }\end{array}$ & $\begin{array}{l}\text { Antibiotic therapy } \\
\text { Steroids may be considered for protracted glomerulonephritis }{ }^{23} \\
\text { Poor prognosis in some cases }\end{array}$ \\
\hline
\end{tabular}

ANCA, anti-neutrophil cytoplasmic antibody; ASO, antistreptolysin O; ASPGN, acute poststreptococcal glomerulonephritis; IEAGN, infective endocarditis-associated glomerulonephritis; MRSA, methicillin-resistant staphylococcus aureus.

lesions may be the first sign of infective endocarditis. In such cases, factors such as anemia, splenomegaly, or purpura can also provide clues to the diagnosis. Moreover, the incidence of acute infective endocarditis has been increasing. According to a 2015 report by Boils et al., acute renal failure was the most common presentation of IEAGN, occurring in $79 \%$ of cases, followed by acute nephritic syndrome in $9 \%$, rapidly progressive glomerulonephritis in $6 \%$, and nephrotic syndrome in $6 \%$ of cases. ${ }^{16}$ It should be noted that, in contrast to conventional APSGN which occurs with nephritis accompanied by abnormal urinary test results, many cases of IEAGN present with acute renal failure (Table 1).

Serological findings reported in this study showed a decrease in complement activity, with reduced $\mathrm{C} 3$ only observed in $37 \%$ of cases and reduced $\mathrm{C} 4$ only in $19 \%$, an additional $16 \%$ had reduced $\mathrm{C} 3$ and $\mathrm{C} 4 .{ }^{16}$ Other reported findings include cryoglobulin and rheumatoid factor. ${ }^{17}$ ANCA-positive cases are also frequently encountered. ${ }^{18}$ In the above study by Boils et al., the ANCA-positive rate was $28 \%$, and proteinase 3-ANCA was significantly more common than myeloperoxidaseANCA. ${ }^{16}$ Other observational studies have reported ANCA-positive rates ranging from $18-33 \%{ }^{19-21}$

Obtaining blood cultures is essential in cases of suspected infective endocarditis. According to the guidelines, diagnosis of infective endocarditis should be established only after obtaining multiple positive blood culture reports. ${ }^{8}$ The causative organism in IEAGN is reported to be Staphylococcus in 53\% of cases and Streptococcus in $23 \% .{ }^{16}$ However, according to Hoen et al., $14 \%$ of cases may have negative blood cultures. ${ }^{22}$ Transesophageal echocardiography is recommended in cases of suspected infective endocarditis, though it has been reported that introducing echocardiography does not reduce the undetected diagnosis rate. ${ }^{23}$ Therefore, diagnosing infective endocarditis requires a comprehensive assessment of clinical and echocardiographic findings, as well as laboratory results.

\section{PATHOLOGY}

Earlier reports on the pathogenesis of IEAGN hypothesized that it was related to embolism, abscess formation, or other lesions arising from bacterial clumps. ${ }^{5}$ However, because of the presence of different histological types such as endocapillary proliferative glomerulonephritis, membranoproliferative glomerulonephritis, and crescentic glomerulonephritis as well as the presence of cases with reduced complement levels and immune complex deposition, the current mainstream thinking is that an immunological mechanism is involved in the development of glomerulonephritis. ${ }^{1}$ Renal lesions due to embolism and abscess formation, like those described in earlier reports on infection-associated glomerulonephritis, are considered to appear in more 

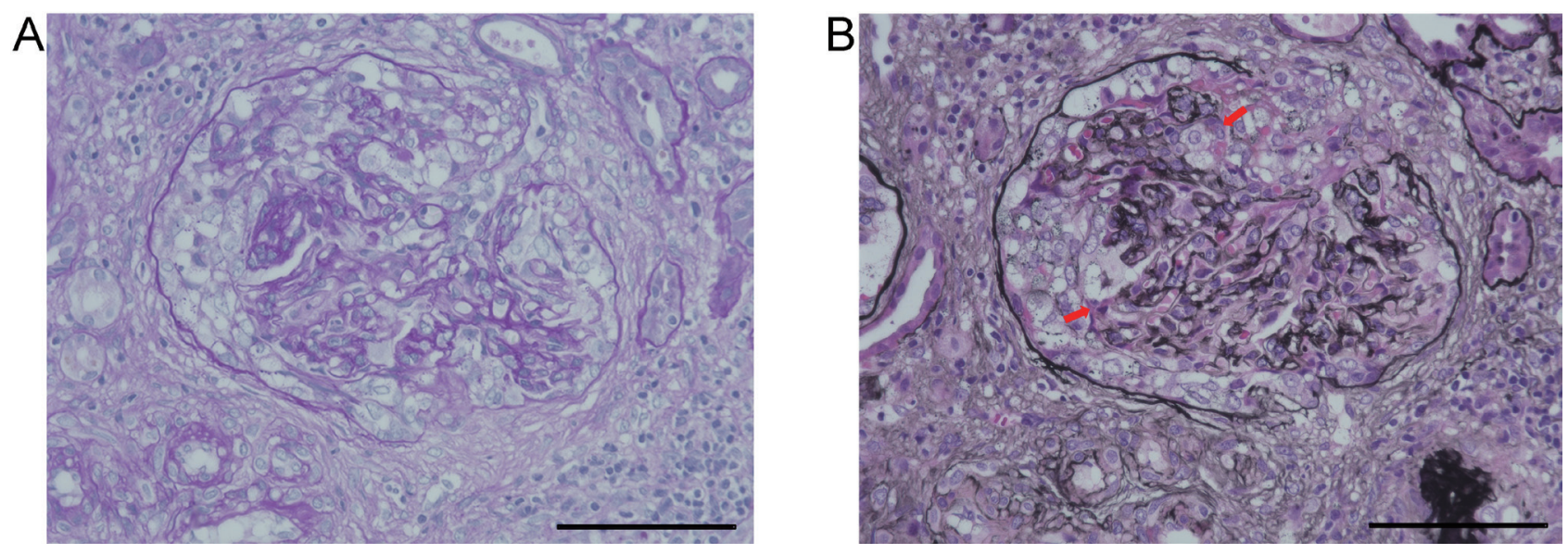

Fig. 2. Representative images of the kidneys in our case (unpublished data). The kidneys were obtained by ultrasound-guided biopsy and fixed with 10\% formaldehyde. The sections were stained with (A) periodic acid-Schiff and (B) periodic acid-methenamine silver. Rupture of the basement membrane (arrows) and cellular crescents were observed in the lumens of Bowman's capsules. Bar $=50 \mu \mathrm{m}$.

advanced stages of the disease, and are more likely to be observed on autopsy. ${ }^{23}$ This could explain why earlier studies reported the presence of these lesions because these studies were often based on autopsy cases. In fact, in a study by Majumdar et al. of 62 cases of IEAGN diagnosed by renal biopsy or autopsy, infarcts were observed in $31 \%$ of cases, all of which were observed on autopsy. ${ }^{24}$ IEAGN related to Staphylococcus aureus, incidence of which has been increasing recently, has various pathological presentations, though crescent formation and necrotizing lesions are often observed on optical microscopy. ${ }^{16}$ It has also been reported that two-thirds of cases have pauci-immune crescentic glomerulonephritis. ${ }^{13,}{ }^{24}$ Endocapillary proliferation is also observed relatively frequently in IEAGN, and is often accompanied by exudative lesions. ${ }^{25}$ In particular, cases with chronic courses, such as subacute infective endocarditis, often present with membranoproliferative glomerulonephritis. In our center, we previously performed a renal biopsy on a 57-year-old male patient with a diagnosis of rapidly progressive glomerulonephritis based on findings of proteinuria, hematuria, and elevated proteinase 3-ANCA levels. Rupture of the basement membrane and cellular crescents were observed in the lumens of Bowman's capsules (Fig. 2, unpublished data). Streptococcus mitis was identified on blood culture and echocardiography showed vegetations. Based on these findings, the patient was diagnosed with IEAGN.

On immunofluorescence, almost all positive cases show involvement of the mesangial area alone and some also show deposition in the basement membrane. The majority are C3-positive, which is thought to reflect immune complex formation. ${ }^{25}$ One study reported that
$44 \%$ of cases exhibited the pauci-immune type staining pattern on immunofluorescence; while on electron microscopy, cases with mesangial deposition were the most common, with $14 \%$ exhibiting hump-like subepithelial deposits. ${ }^{16}$

\section{TREATMENT AND PROGNOSIS}

Identifying the causative organism on blood culture is important for selecting an antibiotic treatment for IEAGN. The methicillin-resistant strain of Staphylococcus aureus (MRSA), which is becoming more virulent, is often identified. Therefore, performing culture and the sensitivity of the culture is important for selecting an appropriate antibiotic therapy. Moreover, the route of invasion and distal foci of infection should be investigated. This facilitates the selection of any necessary surgical treatment, including dental surgery, and/ or switching to a different antibiotic regimen. When cultures are negative, the clinician should decide whether to repeat blood culture or to start empiric therapy with a wide-spectrum antibiotic, while taking into account the patient's general condition. In empiric therapy, antibiotics are selected that provide coverage against the most common causative bacterial species, while paying attention to other aspects such as the onset pattern and patient characteristics. Patients usually require longterm antibiotic therapy, irrespective of causative species, and valve replacement may also be necessary. 8,26

The prognosis for recovery of renal function in IEAGN is poor. Among 83 patients with glomerulonephritis related to Staphylococcus infection, about 50\% experienced persistent nephropathy or kidney failure (remission in 36 cases, persistent renal dysfunction in 15 cases, progression to end-stage renal disease in 19 
Table 2. Clinical manifestations and laboratory findings of AAV and ANCA-positive IRGN ${ }^{28}$

\begin{tabular}{|c|c|c|c|}
\hline & $\begin{array}{l}\text { AAV } \\
n=66\end{array}$ & $\begin{array}{c}\text { ANCA-positive IRGN } \\
n=17\end{array}$ & $P$ value \\
\hline \multicolumn{4}{|l|}{ Clinical manifestations } \\
\hline Sex (male / female) & $31 / 35$ & $5 / 12$ & NS \\
\hline Age, year & $52.8 \pm 11.4$ & $42.5 \pm 17.0$ & $<0.05$ \\
\hline Acute kidney injury, $n(\%)$ & $28(42)$ & $3(18)$ & NS \\
\hline Lung, $n(\%)$ & $33(50)$ & $2(12)$ & $<0.01$ \\
\hline Skin, $n(\%)$ & $16(24)$ & $5(29)$ & NS \\
\hline Splenomegaly, $n(\%)$ & $2(3)$ & $4(23)$ & $<0.05$ \\
\hline New heart murmur, $n(\%)$ & $0(0)$ & $3(18)$ & $<0.01$ \\
\hline Ear/nose/throat, $n(\%)$ & $17(26)$ & $0(0)$ & $<0.05$ \\
\hline Nervous system, $n(\%)$ & $28(42)$ & $1(6)$ & $<0.01$ \\
\hline \multicolumn{4}{|l|}{ Serological parameters } \\
\hline MPO-ANCA, U/mL & $130 \pm 47$ & $166 \pm 38$ & NS \\
\hline PR3-ANCA, U/mL & $93 \pm 14$ & $158 \pm 28$ & NS \\
\hline ANA, $n(\%)$ & $5(7)$ & $5(29 \%)$ & $<0.05$ \\
\hline Anti-cardiolipin antibody, $n(\%)$ & $6(9)$ & $9(53)$ & $<0.001$ \\
\hline Cryoglobulin, $n(\%)$ & $2(3)$ & $8(47)$ & $<0.001$ \\
\hline CRP, mg/dL & $6.7 \pm 3.8$ & $10.5 \pm 2.8$ & NS \\
\hline
\end{tabular}

Modified from reference 28. AAV, ANCA-associated vasculitis; ANA, anti-nuclear antibody; ANCA, anti-neutrophil cytoplasmic antibody; CRP, C-reactive protein; IRGN, infection-related glomerulonephritis; MPO, myeloperoxidase; NS, not significant; PR3, proteinase-3.

cases, death in 12 cases, and 1 case with no record). ${ }^{27}$ Risk factors for poor renal prognosis include older age, diabetes mellitus, renal dysfunction before onset, glomerulosclerosis, and interstitial fibrosis..$^{15}$ Further, heart failure sometimes accompanies infective endocarditis, which can prolong nephropathy. ${ }^{23}$

Differentiating IEAGN from ANCA-associated glomerulonephritis is necessary for the selection of the appropriate treatment plan. Bonaci-Nikolic et al. examined the clinical differences between ANCAassociated vasculitis and ANCA-positive IRGN. They found that the patients with ANCA-positive IRGN were significantly younger and had clinical findings such as the appearance of a heart murmur and splenomegaly. Whereas, hepatomegaly, pulmonary lesions, and neuropathy were significantly more common in ANCA-associated vasculitis (Table 2). ${ }^{28}$ Because ANCA-associated glomerulonephritis sometimes leads to renal dysfunction that requires renal replacement therapy, ${ }^{29}$ extensive immunosuppressive therapies are the mainstream of the therapy. While ANCA-associated glomerulonephritis is treated with steroids and immunosuppressants, immunosuppression should be avoided in IEAGN. In IEAGN with necrotizing lesions or crescent formation, immunosuppression does not improve renal prognosis, rather it is associated with increased mortality. ${ }^{23}$ Steroids may be considered in cases of protracted glomerulonephritis. ${ }^{23}$ In such cases, antibiotic therapy should be administered for the appropriate period to completely eradicate the infection. Renal biopsy can be helpful in assessing the chronicity of glomerulonephritis in uncertain cases.

\section{CONCLUSION}

This review outlined the differences in the clinical presentation of IEAGN and key factors differentiating it from other diseases. In particular, the number of reports on IEAGN that are ANCA positive is increasing, and detailed investigations including blood cultures should be performed in cases that present with acute renal failure. Establishing the diagnosis may be difficult because the pathological picture often resembles the initial presentation of glomerulonephritis occurring due to other factors; therefore, a comprehensive inspection of the case that takes the clinical presentation into account is necessary. 
Acknowledgments: We would like to thank Editage (www. editage.com) for English language editing.

The authors declare no conflict of interest.

\section{REFERENCES}

1 Nasr SH, Radhakrishnan J, D'Agati VD. Bacterial infectionrelated glomerulonephritis in adults. Kidney Int. 2013;83:792803. DOI: 10.1038/ki.2012.407, PMID: 23302723

2 Nadasdy T, Hebert LA. Infection-related glomerulonephritis: understanding mechanisms. Semin Nephrol. 2011;31:369-75. DOI: 10.1016/j.semnephrol.2011.06.008, PMID: 21839370

3 Löhlein M. Ueber hämorrhagische nierenaffektioned bei chronisher ulzerözer endokarditis. Med Klin. 1910;6:375-9.

4 Baehr G. Glomerular lesions of subacute bacterial endocarditis. J Exp Med. 1912;15:330-47. DOI: 10.1084/jem.15.4.330, PMID: 19867526

5 Bell ET. Glomerular lesions associated with endocarditis. Am J Pathol. 1932;8:639-62, 5. PMID: 19970039

6 Durack DT, Lukes AS, Bright DK; Duke Endocarditis Service. New criteria for diagnosis of infective endocarditis: utilization of specific echocardiographic findings. Am J Med. 1994;96:200-9. DOI: 10.1016/0002-9343(94)90143-0, PMID: 8154507

7 Li JS, Sexton DJ, Mick N, Nettles R, Fowler VG Jr, Ryan T, et al. Proposed modifications to the Duke criteria for the diagnosis of infective endocarditis. Clin Infect Dis. 2000;30:6338. DOI: 10.1086/313753, PMID: 10770721

8 Japanese Circulation Society, Japanese College of Cardiology, Japanese Society of Echocardiography, et al. Guidelines for preventing and treating infective endocarditis (revised 2018 edition) [Internet]. Tokyo: The Japanese Circulation Society [cited 2021 Aug]. Available from: https://www.j-circ.or.jp/ cms/wp-content/uploads/2017/07/JCS2017_nakatani_h.pdf.

9 Wilson W, Taubert KA, Gewitz M, Lockhart PB, Baddour LM, Levison M, et al. Prevention of infective endocarditis: guidelines from the American Heart Association: a guideline from the American Heart Association Rheumatic Fever, Endocarditis, and Kawasaki Disease Committee, Council on Cardiovascular Disease in the Young, and the Council on Clinical Cardiology, Council on Cardiovascular Surgery and Anesthesia, and the Quality of Care and Outcomes Research Interdisciplinary Working Group. Circulation. 2007;116:173654. DOI: 10.1161/CIRCULATIONAHA.106.183095, PMID: 17446442

10 Benito N, Miró JM, de Lazzari E, Cabell CH, del Río A, Altclas J, et al.; ICE-PCS (International Collaboration on Endocarditis Prospective Cohort Study) Investigators. Health care-associated native valve endocarditis: importance of nonnosocomial acquisition. Ann Intern Med. 2009;150:586-94. DOI: 10.7326/0003-4819-150-9-200905050-00004, PMID: 19414837

11 Conlon PJ, Jefferies F, Krigman HR, Corey GR, Sexton DJ, Abramson MA. Predictors of prognosis and risk of acute renal failure in bacterial endocarditis. Clin Nephrol. 1998;49:96101. PMID: 9524779

12 Montseny JJ, Meyrier A, Kleinknecht D, Callard P. The current spectrum of infectious glomerulonephritis. Experience with 76 patients and review of the literature. Medicine (Baltimore). 1995;74:63-73. DOI: 10.1097/00005792-19950300000001, PMID: 7891544
13 Nasr SH, Markowitz GS, Stokes MB, Said SM, Valeri AM, D'Agati VD. Acute postinfectious glomerulonephritis in the modern era: experience with 86 adults and review of the literature. Medicine (Baltimore). 2008;87:21-32. DOI: 10.1097/ md.0b013e318161b0fc, PMID: 18204367

14 Wen YK. The spectrum of adult postinfectious glomerulonephritis in the new millennium. Ren Fail. 2009;31:676-82. DOI: 10.3109/08860220903118616, PMID: 19814634

15 Nasr SH, Fidler ME, Valeri AM, Cornell LD, Sethi S, Zoller A, et al. Postinfectious glomerulonephritis in the elderly. J Am Soc Nephrol. 2011;22:187-95. DOI: 10.1681/ASN.2010060611, PMID: 21051737

16 Boils CL, Nasr SH, Walker PD, Couser WG, Larsen CP. Update on endocarditis-associated glomerulonephritis. Kidney Int. 2015;87:1241-9. DOI: 10.1038/ki.2014.424, PMID: 25607109

17 Agarwal A, Clements J, Sedmak DD, Imler D, Nahman NS Jr, Orsinelli DA, et al. Subacute bacterial endocarditis masquerading as type III essential mixed cryoglobulinemia. J Am Soc Nephrol. 1997;8:1971-6. DOI: 10.1681/ASN.V8121971, PMID: 9402102

18 Yoshifuji A, Hibino Y, Komatsu M, Yasuda S, Hosoya K, Kobayashi E, et al. Glomerulonephritis caused by Bartonella spp. infective endocarditis: the difficulty and importance of differentiation from anti-neutrophil cytoplasmic antibodyrelated rapidly progressive glomerulonephritis. Intern Med. 2021;60:1899-906. DOI: 10.2169/internalmedicine.5608-20, PMID: 33456034

19 Ying CM, Yao DT, Ding HH, Yang CD. Infective endocarditis with antineutrophil cytoplasmic antibody: report of 13 cases and literature review. PLoS One. 2014;9:e89777. DOI: 10.1371/journal.pone.0089777, PMID: 24587028

20 Langlois V, Lesourd A, Girszyn N, Ménard JF, Levesque $\mathrm{H}$, Caron F, et al. Antineutrophil Cytoplasmic Antibodies Associated With Infective Endocarditis. Medicine (Baltimore). 2016;95:e2564. DOI: 10.1097/MD.0000000000002564, PMID: 26817911

21 Mahr A, Batteux F, Tubiana S, Goulvestre C, Wolff M, Papo T, et al.; IMAGE Study Group. Brief report: prevalence of antineutrophil cytoplasmic antibodies in infective endocarditis. Arthritis Rheumatol. 2014;66:1672-7. DOI: 10.1002/art.38389, PMID: 24497495

22 Hoen B, Selton-Suty C, Lacassin F, Etienne J, Briançon $\mathrm{S}$, Leport $\mathrm{C}$, et al. Infective endocarditis in patients with negative blood cultures: analysis of 88 cases from a one-year nationwide survey in France. Clin Infect Dis. 1995;20:501-6. DOI: 10.1093/clinids/20.3.501, PMID: 7756467

23 Satoskar AA, Parikh SV, Nadasdy T. Epidemiology, pathogenesis, treatment and outcomes of infection-associated glomerulonephritis. Nat Rev Nephrol. 2020;16:32-50. DOI: 10.1038/s41581-019-0178-8, PMID: 31399725

24 Majumdar A, Chowdhary S, Ferreira MAS, Hammond LA, Howie AJ, Lipkin GW, et al. Renal pathological findings in infective endocarditis. Nephrol Dial Transplant. 2000;15:17827. DOI: 10.1093/ndt/15.11.1782, PMID: 11071965

25 Satoskar AA, Suleiman S, Ayoub I, Hemminger J, Parikh S, Brodsky SV, et al. Staphylococcus infection-associated GN - spectrum of IgA staining and prevalence of ANCA in a single-center cohort. Clin J Am Soc Nephrol. 2017;12:39-49. DOI: 10.2215/CJN.05070516, PMID: 27821389 
26 Baddour LM, Wilson WR, Bayer AS, Fowler VG Jr, Tleyjeh IM, Rybak MJ, et al.; American Heart Association Committee on Rheumatic Fever, Endocarditis, and Kawasaki Disease of the Council on Cardiovascular Disease in the Young, Council on Clinical Cardiology, Council on Cardiovascular Surgery and Anesthesia, and Stroke Council. Infective endocarditis in adults: diagnosis, antimicrobial therapy, and management of complications: a scientific statement for healthcare professionals from the American Heart Association. Circulation. 2015;132:1435-86. DOI: 10.1161/CIR.0000000000000296, PMID: 26373316

27 Wang SY, Bu R, Zhang Q, Liang S, Wu J, Liu XGZSW, et al. Clinical, pathological, and prognostic characteristics of glomerulonephritis related to Staphylococcal infection. Medicine (Baltimore). 2016;95:e3386. DOI: 10.1097/ MD.0000000000003386, PMID: 27082609
28 Bonaci-Nikolic B, Andrejevic S, Pavlovic M, Dimcic Z, Ivanovic B, Nikolic M. Prolonged infections associated with antineutrophil cytoplasmic antibodies specific to proteinase 3 and myeloperoxidase: diagnostic and therapeutic challenge. Clin Rheumatol. 2010;29:893-904. DOI: 10.1007/s10067-0101424-4, PMID: 20306213

29 Mae Y, Takata T, Ida A, Ogawa M, Taniguchi S, Yamamoto M, et al. Prognostic value of neutrophil-to-lymphocyte ratio and platelet-to-lymphocyte ratio for renal outcomes in patients with rapidly progressive glomerulonephritis. J Clin Med. 2020;9:1128. DOI: 10.3390/jcm9041128, PMID: 32326552 